\title{
Interspike Time Distribution in Noise Driven Excitable Systems
}

\author{
A. M. Yacomotti, M. C. Eguia, J. Aliaga, O. E. Martinez, and G. B. Mindlin \\ Departamento de Física, FCEN, UBA, Ciudad Universitaria, Bld. I (1428), Buenos Aires, Argentina
}

A. Lipsich

CEFIS-Instituto Nacional de Tecnología Industrial, P.O. Box 157 (1650), San Martín, Buenos Aires, Argentina

(Received 13 November 1998)

\begin{abstract}
We generate an observable which relates the interspike time statistics in a noise driven excitable system with its phase space global properties. Experimental results from a semiconductor laser with optical feedback are analyzed within this framework.
\end{abstract}

PACS numbers: 42.65.Sf, 05.40.Ca, 05.45.-a, 42.55.Px

Escape problems from metastable states are ubiquitous in nature [1]. From biology to physics, several situations are adequately modeled through noise driven equations for which the dynamical output consists of a sequence of spike responses with a more or less complex interspike time distribution [2,3]. In these cases, efforts usually are devoted to the calculation of rate coefficients. Kramers made the seminal contribution to this program. He computed escape rates from both the local properties of the deterministic part of the model in the neighborhood of the metastable state and the noise level [4]. In Ref. [5], pseudoregular oscillations were found in noise driven excitable systems for a specific case: the infinitely dissipative regime. In this work, we analyze the consequences of the global properties of a general excitable system (presenting finite dissipation) in the interspike time distribution of its response to added noise. We find that the interspike distributions present a nontrivial structure. The global properties we refer to are the stable and unstable manifolds of the fixed points of the deterministic part of the model. In particular, we analyze the results of an experiment (a semiconductor laser with optical feedback close to the onset of a regime called low frequency fluctuations) [6-8], in terms of a simple model [9].

The experimental setup is shown in Fig. 1(a). The diode laser used in our experiment is the single transverse-mode Sharp LT030MD0 (nominal wavelength $\lambda=750 \mathrm{~nm}$; solitary laser threshold $I_{\mathrm{th}}=36.66 \mathrm{~mA}$ ). The temperature of the laser is stabilized to better than $0.01{ }^{\circ} \mathrm{C}$. The beam is collimated and directed toward a high reflection mirror $(R=99 \%)$ located at $50 \mathrm{~cm}$ from the laser, which redirects the beam back to it. An antireflection coated lens ( $f=25 \mathrm{~cm}$ ) is placed within the cavity in order to focus the beam into the mirror, which seems to improve the coupling efficiency. The optical feedback strength is controlled by an acusto-optic modulator (AOM) placed inside the cavity, in such a way that a variable amount of light can be removed from the zero order thus reducing the feedback level. The intensity output is detected by a $1 \mathrm{GHz}$ bandwidth photodiode and the signal is analyzed with a HP 54616B $500 \mathrm{MHz}$ digital oscilloscope. In this work, we are interested in the slow dynamics, i.e., time scales much larger than the external cavity round-trip time $(t \approx 3 \mathrm{~ns})$. Actually, the short-time dynamics are washed out by the use of a $30 \mathrm{MHz}$ low-pass filter. Different dynamical scenarios are observed as the parameters (current, feedback) are varied, which are extensively described in the literature (see [6], and references therein).

For pump values considerably smaller than the solitary laser threshold the laser intensity stays constant in time. For pump values close to the solitary laser threshold the intensity shows pulses (dropouts), with a recovery time that in our experiment is approximately $400 \mathrm{~ns}$, separated by regions of constant intensity from fractions to several microseconds of duration. The last regime $[7,8,10,11]$ is the so-called low frequency fluctuations regime (LFF). A typical time trace is shown in Fig. 1(b). A complete description can be found in [12].

(a)

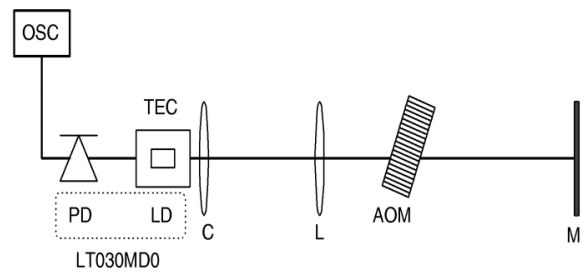

(b)

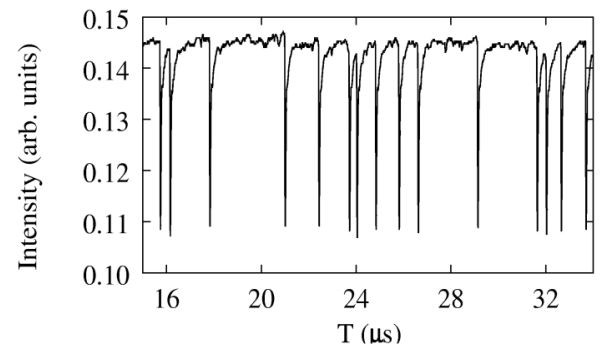

FIG. 1. (a) Experimental setup: LD, laser diode; PD, photodiode; OSC, digitizing oscilloscope; C, collimator; L, lens; M, mirror; AOM, acusto-optic modulator; TEC, thermoelectric cooler. (b) Time trace of the intensity as a function of time in what is called low frequency fluctuations (LFF) regime, displaying some clusters. $I=37.63 \mathrm{~mA} ; V_{\mathrm{AOM}}=0 \mathrm{~V}$. 
In this work we deal with the statistics of time intervals between dropouts, which are determined by digitizing and using a peak-detecting algorithm until $10^{4}$ peaks are recorded. With maximum feedback (AOM voltage, $\left.V_{\mathrm{AOM}}=0 \mathrm{~V}\right)$ we obtain a threshold reduction $\left(I_{\mathrm{th}}-\right.$ $\left.I_{\text {ext }}\right) / I_{\text {th }}=0.11$, where $I_{\text {th }}$ and $I_{\text {ext }}$ stand for the solitary laser threshold current and the external-cavity laser threshold current, respectively. Approximately $80 \%$ of the emitted light is reflected back toward the laser. For AOM voltage $V_{\mathrm{AOM}}=9.8 \mathrm{~V}$ the amount of feedback is reduced by $5 \%$ and the threshold reduction is lowered for about $10 \%$. All the measurements were made with the same alignment conditions of the external mirror. In this situation, the distribution of time intervals shows a doublepeaked structure (Fig. 2). The left side peak corresponds to consecutive dropouts that constitute what are usually called "clusters" [Fig. 1(b)]. The right side peak corresponds to statistically distributed events (i.e., long term exponential decay) [7].

Figure 2(a) shows a family of histograms for different pumping currents and no voltage in the AOM. As the pumping current is increased from 37.22 to $37.91 \mathrm{~mA}$, the peaks at the right are higher, centered at smaller time
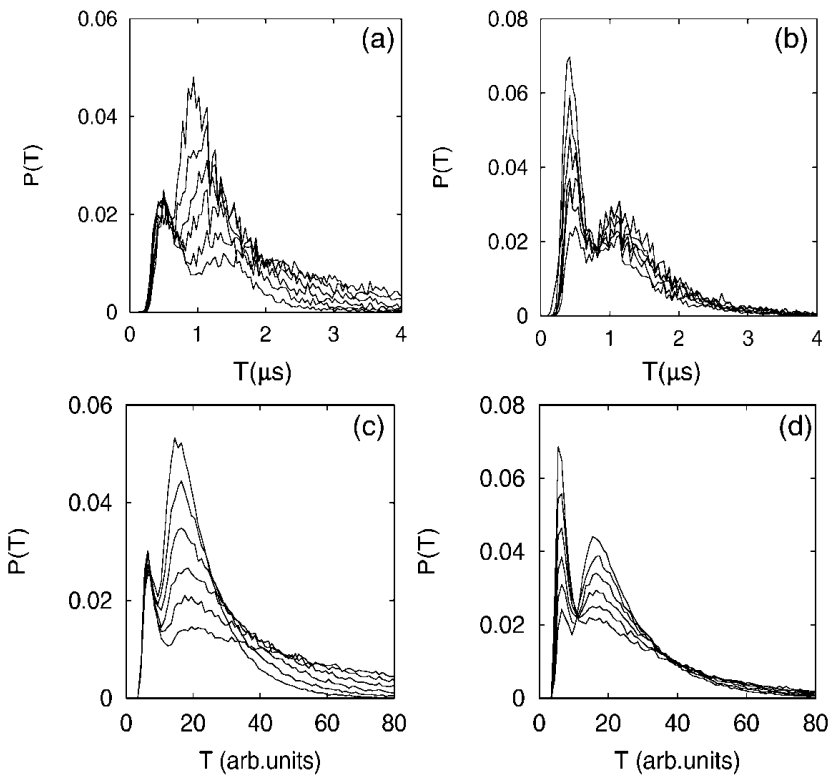

FIG. 2. Probability distributions of time between dropout events in the laser experiment (a),(b) and in the model (c),(d). Experimental histograms (with a bin size of $0.04 \mu \mathrm{s}$ ): (a) $V_{\mathrm{AOM}}=0 \mathrm{~V}$ and different current values (in $\mathrm{mA}$ ): 37.22 , $37.36,37.49,37.63,37.77$, and 37.91 , corresponding to increasing rightmost peak amplitudes; (b) $I=37.63 \mathrm{~mA}$ and different $V_{\text {AOM }}$ values (in $\mathrm{V}$ ): $0,5.8,6.8,7.8,8.8$, and 9.8 , corresponding to increasing leftmost peak amplitudes. Interspike histograms of the $x$ variable for the system described by Eqs. (1) and (2) (with a bin size of 1 a.u.): (c) $\epsilon_{2}=0.5$ and different values of $\epsilon_{1}: 0.22,0.23,0.24,0.25,0.26$, and 0.27 , corresponding to increasing rightmost peak amplitudes; (d) $\epsilon_{1}=0.25$ and different $\epsilon_{2}$ values: $0.5,0.48,0.46,0.44,0.42$, and 0.4 , corresponding to increasing leftmost peak amplitudes. values, and decay faster. In addition, the peaks at the left do not change appreciably, neither in height nor in location. Figure 2(b) depicts a family of histograms for different AOM voltage and $I=37.63 \mathrm{~mA}$. In this case, the peaks do not change their location, but their relative heights do. The exponential decay of the rightmost peaks remains almost unchanged. The two characteristic times of the interspikes can directly be observed in the time series data [Fig. 1(b)]. As the feedback is decreased, the clusters contain more pulses. Inspecting the time series we check that these two characteristic times are present for several alignment conditions (the optimum one among them). The results reported in this work correspond to an alignment that enhances the resolution of the peaks in the histograms.

Let us explore a dynamical model which could account for these observations. It was recently proposed, based on experimental observations, that the semiconductor laser with feedback behaves as an excitable system within the LFF regime [6]. Excitability was recently also found in a widely used model for the phenomenon [10]. It is a typical strategy in nonlinear dynamics to find paradigmatic equations (if possible the simplest ones) presenting families of solutions with a desired feature. To study excitable behavior, it is possible to analyze the behavior of a system close in parameter space of an Andronov bifurcation [13]. In such a case, three fixed points coexist: a repulsor, a saddle, and a node. Their manifolds are organized as follows: the unstable manifold of the saddle is the stable manifold of the node, and the saddle is connected through its stable manifold with the repulsor (see Fig. 3). The equations we are studying are [9]

$$
\begin{aligned}
& x^{\prime}=y, \\
& y^{\prime}=x-y-x^{3}+x y+\epsilon_{1}+\epsilon_{2} x^{2},
\end{aligned}
$$

with $(x, y) \in R^{2}$, and $\epsilon_{1}, \epsilon_{2} \in R^{+}$. Within the excitable regime (region II of Fig. 3), an initial condition close to the node, subject to the action of noise, may cross the stable manifold of the saddle to relax after a long excursion in phase space. We associate this pulse with a dropout event of the laser intensity. In order to obtain excitable pulses in our model, we added a Gaussian white noise term in Eq. (1) with zero mean and variance $D=10^{-3}$.

Now, we will focus on the study of the interspike time distributions in our model. The rate of escape from the node is ruled only by local properties, and can be described in terms of Kramers' formula [1,4]. The theory also predicts a wide peak in the probability distribution, with a fast rise for short times and an exponential tail for the longer ones. Usually, for excitable systems, it is assumed that spikes are triggered only from a quiescent state. However, in our model it is possible to have an early-triggered spike if a trajectory crosses the stable manifold of the saddle at some point before reaching the node. In such a case the interspike time equals the time of flight of a trajectory following the unstable manifold of 


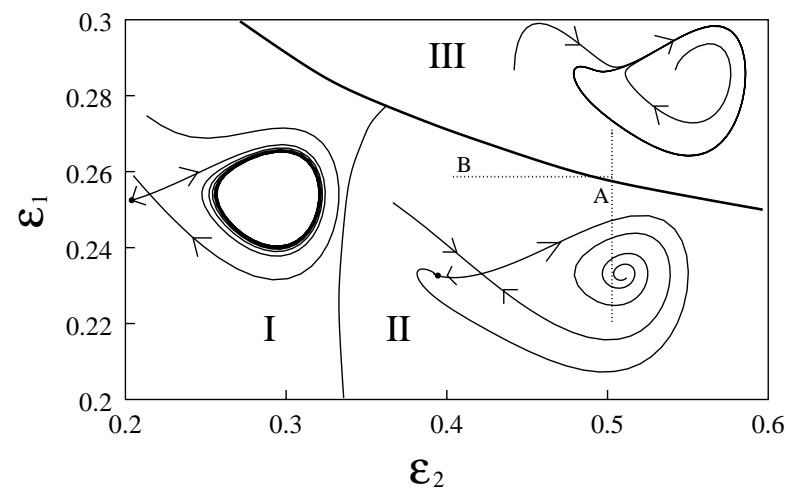

FIG. 3. Bifurcation diagram and phase portraits for the system described by equations in the text. In regions I and II there are three fixed points: a node, a saddle, and a repulsor. Crossing the separatrix to region III, the saddle and the attractor collapse. The two lower regions display a qualitatively different behavior. In region I the unstable manifold of the saddle approaches a limit cycle. In region II the unstable manifold of the saddle is the stable manifold of the node and the system behaves as an excitable one. Dotted lines indicate the values at which the histograms of Fig. 2 are obtained: $A$ corresponds to Fig. 2(c) and $B$ corresponds to Fig. 2(d).

the saddle. This second characteristic time superimposes another peak in the histogram. Then we are able to reproduce the bimodal distributions observed in the experiment (see Fig. 2).

In agreement with the experimental observations, the peak on the left is associated with clusters of dropout events and the other peak to much slower escapes from a quiescent state. From a geometrical point of view, the leftmost peak depends on the distance between the manifolds of the saddle (the unstable which guides the trajectory and the stable which acts as a threshold) [14], while the rightmost peak is related to the distance between the saddle and the node. This correspondence can be seen in the phase portrait displayed in Fig. 4.

We now study how the histograms are varied as we move the two parameters. From Fig. 3 it can be seen that the approach to the Andronov bifurcation can be controlled with $\epsilon_{1}$, while the distance to a saddle loop global bifurcation can be controlled by changing $\epsilon_{2}$. Thus, our first parameter is the main one responsible for the distance between the fixed points and consequently the exponential decay of the second peak. As $\epsilon_{1}$ is raised the escapes from the node become faster and the peak grows. The peak at the left remains almost unchanged. This behavior is exactly what is observed in the laser experiment as the current is varied [compare Figs. 2(a) and 2(c)].

On the other hand, $\epsilon_{2}$ affects the distance between manifolds and therefore it also affects the probability of an early crossing. For a fixed escape rate from the node, as we decrease the value of $\epsilon_{2}$, the probability of cluster production increases. Hence, the first peak in the histogram increases at the expense of the second one. Again, we easily repro-

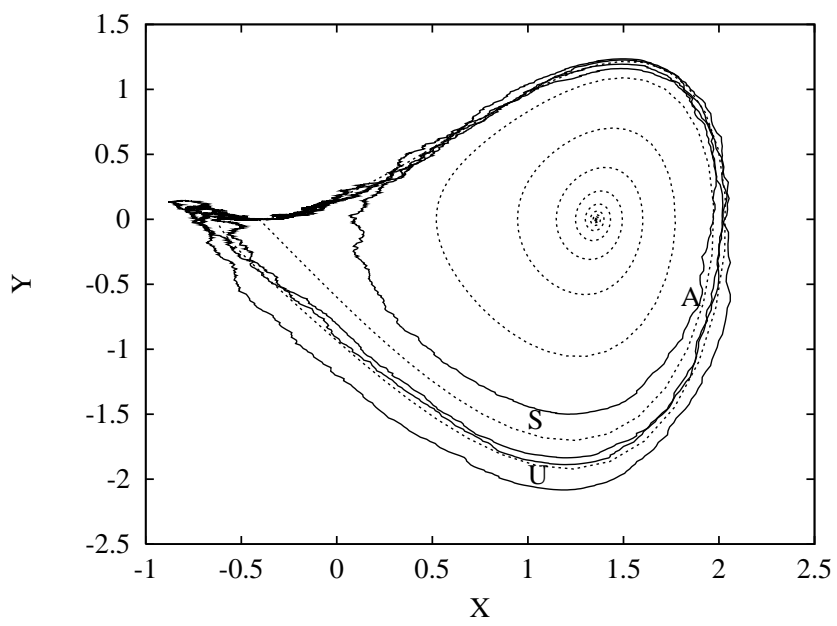

FIG. 4. Phase portrait for the system described by Eqs. (1) and (2) with added noise and parameter values: $\epsilon_{1}=0.26$ and $\epsilon_{2}=0.44$. The dotted lines show the stable $(S)$ and unstable $(U)$ manifolds of the deterministic saddle and the solid line is a portion of trajectory obtained when noise is added. We show four spikes, three of which are originated from escapes from the node and one early-triggered spike $(A)$ from a crossing over the stable manifold of the saddle. This last spike corresponds to one "cluster" and adds one count on the leftmost peak of the histogram.

duce the experimental results varying the amount of feedback. This is displayed in Figs. 2(b) and 2(d).

Note that the bimodal distribution holds even after the Andronov bifurcation (region III of Fig. 3) takes place. This can be understood by noting that a region of slow dynamics arises as a scar of the saddle node bifurcation in the limit cycle. The two peaks correspond to trajectories reinjected before and after this slow dynamics region. Also in region I we can observe two characteristic times, but the inspection of the time series allows us to distinguish it from region II through the presence of large clusters corresponding to visits to the limit cycle.

Noise driven excitable systems can display complex time evolutions. Yet, the validation of such a scenario is by no means trivial. Indirect tests have been performed [6] that consist in the inspection of the response of the system to a periodic forcing. Instead, we decided to assume excitability and compare the evolution of the histograms of the interspike times with what is observed in a minimal model. The remarkable agreement between our dynamical caricature and the experiment builds confidence on the excitable plus noise model.

In this work we analyze the interspike time distribution in noise driven excitable systems. In particular, we study a system with an attractor, a saddle, and a repeller. We relate the statistical features of its solutions with the phase space organization of the invariant manifolds of the fixed points of the noise-free system. It is well understood that an initial condition in the neighborhood of the deterministic node can eventually cross a barrier (in our case, the stable 
manifold of a deterministic saddle) when the system is subjected to noise. What we show now is that noise can play a crucial role in the behavior of the system before it returns to the initial neighborhood. In this case, the global shape of the invariant manifolds can qualitatively alter the interspike histograms, leaving there their fingerprints. As we have shown, the phenomenon can be observed even when the thresholds are not given by manifolds but their scars. This allows us to claim that this effect can be also observed in more general excitable systems with one fixed point, as long as the phase space is preparing a saddle repeller bifurcation [15] in the neighborhood of the attractor.

We thank Jorge Alvarez (UBA) and S. Ponce Dawson (UBA) for helpful discussions, and P. Holmes (Princeton University) for providing us with useful references and comments. This work has been partially funded by Fundación Antorchas, CONICET, Agencia, and UBA.

[1] P. Hanggi, P. Talkner, and M. Borkovec, Rev. Mod. Phys. 62, 251 (1990).

[2] F. Julicher, A. Ajdari, and J. Prost, Rev. Mod. Phys. 69, 1269 (1998).
[3] L. Gammaitoni, P. Hanggi, P. Jung, and F. Marchesoni, Rev. Mod. Phys. 70, 223 (1998).

[4] H. Kramers, Physica (Utrecht) 7, 284 (1940).

[5] D. Sigeti and W. Horsthemke, J. Stat. Phys. 54, 1217 (1989).

[6] M. Giudici, C. Green, G. Giacomelli, U. Nespolo, and J. Tredicce, Phys. Rev. E 55, 6414 (1997).

[7] D. W. Sukow, J. R. Gardner, and D. J. Gauthier, Phys. Rev. A 56, R3370 (1997).

[8] G.H.M. van Tartwijk, A. M. Levine, and D. Lenstra, IEEE J. Sel. Top. Quantum Electron. 1, 466 (1995); I. Fischer, G. H. M. van Tratwijk, A. M. Levine, W. Elsässer, E. O. Göbel, and D. Lenstra, Phys. Rev. Lett. 76, 220 (1996).

[9] M. C. Eguia, G. B. Mindlin, and M. Giudici, Phys. Rev. E 58, 2636 (1998).

[10] J. Mulet and C. R. Mirasso (to be published).

[11] A. Hohl, H. J. C. van der Linden, and R. Roy, Opt. Lett. 20, 2396 (1995).

[12] T. Heil, I. Fischer, and W. Elsasser, Phys. Rev. A 58, R2672 (1998).

[13] P. C. Coullet, S. C. Müller, and D. Walgraef, Chaos 4, 3 (1994).

[14] E. Stone and P. Holmes, SIAM J. Appl. Math. 50, 726 (1990).

[15] S. Ponce Dawson and J. Pearson (to be published). 